\title{
Retrospective use of heparin for VTE prophylaxis in adult patients on hemodialysis
}

\author{
Kasie Stephens $^{1 *}$ and Tramaine Young ${ }^{2}$ \\ ${ }^{1}$ PharmD candidate, UNC REX Healthcare, Raleigh, USA \\ ${ }^{2}$ PharmD, MSCR, BCPS Inpatient Pharmacy, UNC REX Healthcare, Raleigh, USA
}

\begin{abstract}
Background: There is minimal research on whether heparin 5000 units subcutaneous (Sub-q) should be dosed every 8 hours versus every 12 hours in hemodialysis (HD) patients for venous thromboembolism (VTE) prophylaxis.

Methods: Management of the research data for this study involved collection, entry, processing, storage, retrieval, archival, distribution and documentation of information collected according to a written protocol. Efficacy and safety were measured using a Fisher's exact test with a two-tailed p-value. A p-value of less than 0.05 was considered significant.
\end{abstract}

Study population: An EPIC report was obtained for hemodialysis patients that received heparin 5000 units Sub-q every 8 hours or every 12 hours from March 13 , 2014 to April 28, 2020. 4303 patients were included, and 273 patients were assessed for eligibility. 214 patients were analyzed.

Results: The primary endpoint-vascular event, ischemic event, death related to coagulopathy, anaphylaxis, hyperkalemia, hypertriglyceridemia, heparin induced thrombocytopenia (HIT), or sepsis in patients receiving heparin who were on HD occurred in 12 of 107 patients (11.2\%) in the heparin 5000 units Sub-q every 8 hours group and 10 of 107 patients (9.3\%) in the heparin 5000 units Sub-q every 12 hours group ( $\mathrm{p}=0.676)$.

Conclusion: In HD patients on heparin for VTE prophylaxis, we detected no statistically significant difference between heparin 5000 units Sub-q every 8 hours versus every 12 hours.

\section{Introduction}

Unfractionated heparin (UFH) is indicated for the prophylaxis and treatment of thromboembolic disorders such as venous thromboembolism, acute coronary syndrome, as well as the prevention of clotting during dialysis. UHF works by inhibiting thrombin, which prevents fibrin formation and thrombin activation of platelets and clotting factors $\mathrm{V}$ and VIII. For UHF to exert its effect it requires cofactor antithrombin-III (AT-III) and a minimum of 13 additional saccharide units [1]. The heparin/AT-III complex increases thrombin inhibition by up to 1,000 times [2]. This binding facilitates the inactivation of clotting factors IIa, Xa, IXa, and XIIa [1]. UFH can be administered as a continuous intravenous (IV) infusion or subcutaneous injection as it cannot be absorbed through the gastrointestinal mucosa [3].

In HD patients, the clotting cascade is activated when blood components make contact with the dialyzer, dialysis tubing or the drip chamber [4]. Upon contact, platelet adherence occurs within the circuit, accelerating thrombin generation through the intrinsic coagulation pathway [5]. Due to the increased risk of clotting and thrombosis, anticoagulation is usually required. UFH has been extensively studied throughout the years and has been the standard anticoagulant due to it's favorable side effect profile and safety data in patients who are not experiencing active bleeding, thrombocytopenia, heparin allergy or HIT.

Clots within the dialyzer decrease the surface area of the dialyzer, and in some cases can block blood in the circuit. Historically, the dose of UFH was based off of doses sufficient enough to cause a rise in activated partial thromboplastin time (aPTT) or whole blood activated clotting time (ACT) [4]. Dosing based off of aPTT and ACT is now considered impractical. Today, loading doses and infusion rates are calculated based off of equations to predict UFH dosages. UFH, with a half-life of 1.5 hours is typically given as a loading dose of 1000-2000 international units that is followed by a continuous infusion of 500-1500 units/h [5]. Some centers prescribe an empirical dose of UFH with a loading dose (LD) between 25-75 international units/kg, followed by a maintenance dose (MD) of 500-1500 units/h [6]. Whereas, other centers prefer a larger dose of UFH at the start of dialysis, followed by a second smaller dose halfway through HD [6]. In practice, the bolus dose, infusion rate and stopping times of UHF are determined empirically, based off of patient specific clot formation. The UHF dose might need to be adjusted in patients with a high hematocrit, thrombocytopenia or in patients on long-term anticoagulation [5].

In the practice setting, there is no standard dosing for heparin in HD patients. Due to this, there is minimal research on whether heparin 5000 units Sub-q should be dosed every 8 hours versus every 12 hours in HD patients. This cohort will provide the best available evidence on

${ }^{\star}$ Correspondence to: Kasie Stephens, PharmD candidate, UNC REX Healthcare, Raleigh, USA, E-mail: klstephens1218@email.campbell.edu

Key words: venous thromboembolism, hemodialysis, unfractionated heparin, clotting factors

Received: November 26, 2020; Accepted: December 04, 2020; Published: December 07, 2020 
safety and efficacy in hemodialysis patients utilizing heparin 5000 units Sub-q every 8 hours versus every 12 hours for VTE prophylaxis.

\section{Methods}

\section{Trial design}

We conducted a retrospective, randomized, quality-improvement cohort to evaluate the use of heparin for VTE prophylaxis in hemodialysis patients at UNC REX Healthcare System.

Management of the research data for this study involved collection, entry, processing, storage, retrieval, archival, distribution and documentation of information collected according to a written protocol. An EPIC report was obtained for hemodialysis patients that received heparin 5000 units Sub-q every 8 hours or every 12 hours from March 13, 2014 to April 28, 2020.

Anaphylaxis was defined as a life-threatening allergic reaction that can be characterized by difficulty breathing, shock and dermatologic rash [7]. Hyperkalemia was defined as an elevated serum potassium level greater than $5.5 \mathrm{mEq} / \mathrm{L}$ [8]. Hypertriglyceridemia was defined as triglycerides greater than $150 \mathrm{mg} / \mathrm{dL}$ in the blood [9]. Heparin-induced thrombocytopenia was defined as a life-threatening hypercoagulable state 5-10 days after exposure to heparin therapy [10]. Sepsis was defined as life-threatening organ dysfunction due to infection that overwhelms the host's defense system [11]. Major bleed was defined by The Thrombolysis in Myocardial Infarction (TIMI) bleeding criteria [12].

\section{Trial population}

Participants were included if they were greater than or equal to 18 years of age, received hemodialysis therapy in the UNC REX Healthcare System, received unfractionated heparin therapy, and was a Medicare primary payer.

\section{Trial endpoints}

The primary endpoint compared the incidence of patients experiencing any of the following during the study period: symptomatic or asymptomatic vascular event (VTE (deep vein thrombosis (DVT) or pulmonary embolism (PE)) and/or PE, or acute coronary syndrome (ACS), ischemic event (Stroke, transient ischemic attack (TIA), or Myocardial Infarction(MI), death related to coagulopathy, anaphylaxis, hyperkalemia, hypertriglyceridemia, heparin-induced thrombocytopenia and sepsis.

The secondary endpoint compared the safety incidence events of VTE prophylaxis with both dosing regimens, including patients experiencing a major bleed (bleeding due to unscheduled medical intervention, healthcare professional intervention, impairment of daily living, and/or need to alter medication dosing).

\section{Statistical analysis}

Efficacy and safety in treatment arms was measured using a Fisher's exact test with a two-tailed p-value. A P-value of less than 0.05 was considered significant.

Categorical variables such as demographic characteristics and medical history data were summarized with the use of frequencies and proportions.

\section{Results}

From March 2014 through April 2020, 4303 patients were included, and 273 patients were assessed for eligibility. 214 patients were analyzed from the UNC REX Healthcare System in North Carolina (Figure.1). The baseline characteristics of the patients are listed in Table 1 .

\section{Endpoints}

The primary endpoint-vascular event, ischemic event, death related to coagulopathy, anaphylaxis, hyperkalemia, hypertriglyceridemia, HIT, or sepsis in patients receiving heparin who were on HD occurred in 12 of 107 patients (11.2\%) in the heparin 5000 units Sub-q every 8 hours group and 10 of 107 patients $(9.3 \%)$ in the heparin 5000 units Sub-q every 12 hours group $(\mathrm{p}=0.676)$.

The incidences of the individual components of the primary endpoint are shown in Table 2. Vascular events occurred in 2 of 107 patients (1.9\%) in the heparin 5000 units Sub-q every 12 hours group and 1 of 107 patients $(0.9 \%)$ in the heparin 5000 units Sub-q every 8 hours group $(p=1)$.

\section{Patients were assessed for eligibility}

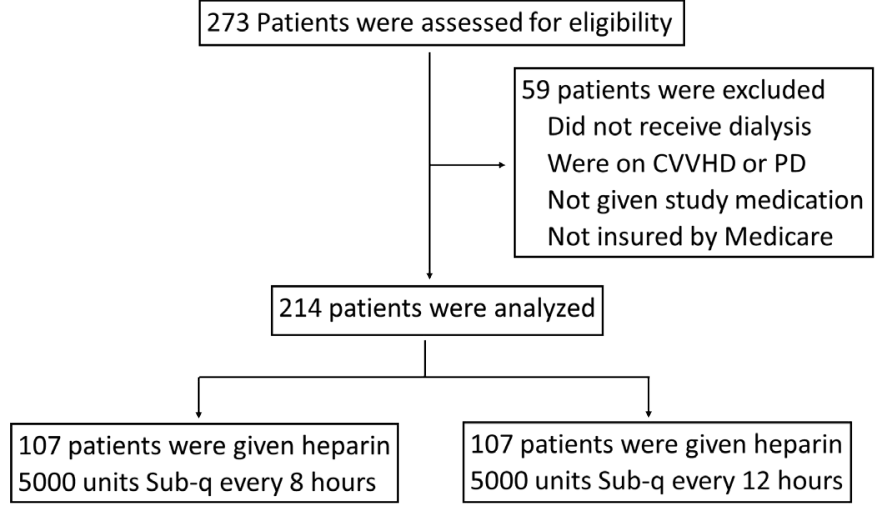

Figure 1. Trial end points

Table 1. Baseline demographics

\begin{tabular}{|c|c|}
\hline Baseline demographics & All patients $(\mathrm{N}=4303)$ \\
\hline Mean age, years & 61.2 \\
\hline Male, n (\%) & $2244(52.1)$ \\
\hline Female, n (\%) & $2059(47.9)$ \\
\hline \multicolumn{2}{|l|}{ Race, $\mathbf{n}(\%)$} \\
\hline White & $1530(35.5)$ \\
\hline Asian & $38(1.0)$ \\
\hline African American & $2503(58.2)$ \\
\hline American Indian or Alaska Native & $28(0.7)$ \\
\hline Other & $200(4.6)$ \\
\hline \multicolumn{2}{|l|}{ Ethnicity, n (\%) } \\
\hline Hispanic or Latino & $130(3.0)$ \\
\hline Non-Hispanic or Latino & $4099(95.2)$ \\
\hline Unknown & $72(1.7)$ \\
\hline \multicolumn{2}{|l|}{ Smoking Status, n (\%) } \\
\hline Former Smoker & $1568(36.4)$ \\
\hline Non-Smoker & $1658(38.5)$ \\
\hline Current every day Smoker & $508(11.8)$ \\
\hline Never Assessed & $135(3.1)$ \\
\hline \multicolumn{2}{|l|}{ Smokeless Tobacco Use, n (\%) } \\
\hline Former User & $160(3.7)$ \\
\hline Never Used & $2741(63.7)$ \\
\hline Current User & $82(1.9)$ \\
\hline Unknown & $1058(25.1)$ \\
\hline \multicolumn{2}{|l|}{ Disease State } \\
\hline Hypertension, n (\%) & $2454(57.0)$ \\
\hline Hyperlipidaemia, n (\%) & $739(17.2)$ \\
\hline Type II Diabetes, n (\%) & $203(4.7)$ \\
\hline Other, $\mathrm{n}(\%)$ & $907(21.1)$ \\
\hline
\end{tabular}


Table 2. Clinical end points

\begin{tabular}{|c|c|c|c|}
\hline End points & $\begin{array}{l}\text { Heparin } 5000 \text { units Sub-q every } 8 \text { h } \\
\qquad(N=107)\end{array}$ & $\begin{array}{l}\text { Heparin } 5000 \text { units Sub-q every } 12 \text { h } \\
\qquad(N=107)\end{array}$ & p-value \\
\hline Primary outcome, n (\%) & $12(11.2)$ & $10(9.3)$ & 0.676 \\
\hline - Vascular event, n (\%) & $1(0.9)$ & $2(1.9)$ & 1 \\
\hline -VTE (DTE, PE) & $0(0.0)$ & $0(0.0)$ & 0 \\
\hline -ACS (UA, NSTEMI, STEMI) & $1(0.9)$ & $2(1.9)$ & 1 \\
\hline • Ischemic event, n (\%) & $1(0.9)$ & $3(2.8)$ & 0.624 \\
\hline -Stroke & $0(0.0)$ & $1(0.9)$ & 1 \\
\hline -TIA & $1(0.9)$ & $0(0.0)$ & 1 \\
\hline -MI & $0(0.0)$ & $2(1.9)$ & 0.498 \\
\hline - Death related to coagulopathy, n (\%) & $0(0.0)$ & $0(0.0)$ & 0 \\
\hline • Anaphylaxis, n (\%) & $1(0.9)$ & $0(0.0)$ & 1 \\
\hline • Hyperkalaemia, n (\%) & $2(1.9)$ & $5(4.7)$ & 0.282 \\
\hline - Hypertriglyceridemia, n (\%) & $4(3.7)$ & $3(2.8)$ & 0.723 \\
\hline$\bullet \mathrm{HIT}, \mathrm{n}(\%)$ & $2(1.9)$ & $0(0.0)$ & 0.498 \\
\hline - Sepsis, n (\%) & $1(0.9)$ & $0(0.0)$ & 1 \\
\hline Secondary outcome, n (\%) & $3(2.8)$ & $4(3.7)$ & 1 \\
\hline • Major bleed & $1(0.9)$ & $0(0.0)$ & 1 \\
\hline - Bleed requiring medical intervention & $0(0.0)$ & $0(0.0)$ & 0 \\
\hline - Bleed requiring healthcare intervention & $1(0.9)$ & $4(3.7)$ & 0.369 \\
\hline - Bleed causing impairment of daily living & $0(0.0)$ & $0(0.0)$ & 0 \\
\hline - Bleed requiring alternative dosing & $1(0.9)$ & $0(0.0)$ & 1 \\
\hline
\end{tabular}

VTE: Venous thromboembolism; DVT: Deep vein thrombosis; PE: Pulmonary embolism; ACS: Acute coronary syndrome; UA: Unstable angina; NSTEMI: non-ST elevation myocardial infarction; STEMI: ST elevation myocardial infarction; TIA: Transient ischemic attack; MI: Myocardial infarction; HIT: Heparin induced thrombocytopenia

ACS occurred in 2 of 107 patients (1.9\%) in the heparin 5000 units Sub-q every 12 hours group and 1 of 107 patients $(0.9 \%)$ in the heparin 5000 units Sub-q every 8 hours group $(p=1)$. Ischemic events occurred in 3 of 107 (2.8\%) patients in the heparin 5000 units Sub-q every 12-hour group and 1 of 107 (0.9\%) patients in the heparin 5000 units Sub-q every 8-hour group ( $\mathrm{p}=0.624)$. Myocardial infarction (MI) occurred in 2 of 107 patients (1.1\%) in the heparin 5000 units Sub-q every 12 hour group and 0 of 107 patients $(0.0 \%)$ in the heparin 5000 units Sub-q every 8 hour group $(\mathrm{p}=0.498)$. Hyperkalemia occurred in 5 of 107 patients (4.7\%) in the heparin 5000 units Sub-q every 12 hours group and 2 of 107 patients (1.9\%) in the heparin 5000 units Sub-q every 8 -hour group ( $\mathrm{p}=0.282$ ). Hypertriglyceridemia occurred in 3 of 107 patients $(2.8 \%)$ in the heparin 5000 units Sub-q every 12hour group and 4 of 107 patients (3.7\%) in the heparin 5000 units Sub-q every 8 -hour group $(\mathrm{p}=0.723)$. HIT occurred in 2 of 107 patients (1.9\%) in the heparin 5000 units Sub-q every 8-hour group and 0 of 107 patients $(0.0 \%)$ in the heparin 5000 units Sub-q every 12-hour group $(\mathrm{p}=1)$.

The secondary endpoint-major bleed, bleed requiring medical intervention, bleed requiring healthcare intervention, bleed causing impairment of daily living, or bleed requiring alternative dosing, occurred in 3 out of 107 patients (2.8\%) in the heparin 5000 units Sub-q every 8 hours group and 4 out of 107 patients (3.7\%) in the heparin 5000 units Sub-q every 12 hours group $(\mathrm{p}=1)$. Bleeding requiring healthcare intervention occurred in 4 of 107 patients (3.7\%) in the heparin 5000 units Sub-q every 12 hour group and 1 of 107 patients $(0.9 \%)$ in the heparin 5000 units Sub-q every 8 hour group $(\mathrm{p}=0.369)$.

\section{Discussion}

Groups were relatively similar between the treatment arms. No accounts of VTE, death related to coagulopathy, bleed requiring medical intervention or bleed causing impairment of daily living occurred in either treatment arm. No statistical difference was detected between either the primary endpoint or the secondary endpoint. There was a higher degree of vascular events, ischemic events and MI in patients in the heparin 5000 units Sub-q every 12 hours treatment arm. One weakness of this study was the small population size of 214 patients. Given more patients, a statistical difference may have been detected between the two groups. Two out of 107 patients every 8-hour group developed HIT. The occurrence of HIT was not seen in every 12-hour group. The incidence of bleeding was similar between both groups.

\section{Conclusion}

In conclusion, groups were relatively similar between the treatment arms. No accounts of VTE, death related to coagulopathy, bleed requiring unscheduled medical intervention or bleed leading to impairment of daily living occurred in either treatment arm. No statistical difference was detected between either the primary endpoint or the secondary endpoint. There was a two or less occurrence difference in all endpoints except for hyperkalemia ( 2 vs. 5 ) and bleed requiring healthcare professional intervention (1 vs. 4 ) in patients in the heparin 5000 units Sub-q every 12 hours treatment arm.

\section{Acknowledgement}

The project described was supported by North Carolina Translational and Clinical Sciences Institute. The content is solely the responsibility of the authors and does not necessarily represent the official views of NC TraCS.

\section{Disclosures}

Authors of this presentation have nothing to disclose concerning possible financial or personal relationships with commercial entities that may have a direct or indirect interest in the subject matter of this presentation.

\section{References}

1. Shaughnessy SG, Warkentin TE, Hirsh J (2001) Heparin and low-molecular weight heparin: mechanisms of action, pharmacokinetics, dosing, monitoring, efficacy, and safety. Chest 119: 64S-94S.

2. Damus PS, Hicks M, Rosenberg RD (1973) Anticoagulant action of heparin. Nature 246: 355-357. 
3. Hull RD, Raskob GE, Hirsh J, Jay RM, Leclerc JR, et al. (1986) Continuous intravenous heparin compared with intermittent subcutaneous heparin in the initial treatment of proximal-vein thrombosis. $N$ Engl J Med 315: 1109-1114.

4. Cronin RE, Reilly RF (2010) Unfractionated heparin for hemodialysis: still the best option. Semin Dial 23: 510-515.

5. Ashby D, Borman N, Burton J, Corbett R, Davenport A, et al. (2019) Renal Association Clinical Practice Guideline of Hemodialysis. BMC Nephrology 20: 379.

6. Murea M, Russell GB, Daeihagh P, Saran AM, Pandya K, et al. (2018) Efficacy and safety of low-dose heparin in hemodialysis. Hemodial Int 22: 74-81.

7. Arnold JJ, Williams PM (2011) Anaphylaxis: Recognition and Management. Am Fam Physician 84: 1111-1118.
8. Lehnhardt A, Kemper MJ (2011) Pathogenesis, diagnosis and management of hyperkalemia. Pediatr Nephrol 26: 377-384.

9. Grundy SM, Stone NJ, Bailey AL (2019) 2018 AHA/ ACC/AACVPR/AAPA/ABC/ ACPM/ADA/AGS/ APhA/ASPC/NLA/PCNA Guideline on the Management of Blood Cholesterol. Circulation 139: e1082-e1143.

10. Cuker A, Arepally GM, Chong BH, Cines DB, Greinacher A, et al. (2018) American Society of Hematology 2018 guidelines for management of venous thromboembolism: heparin-induced thrombocytopenia. Blood Adv 27: 3360-3392.

11. Rhodes A, Evans LE, Alhazzani W, Levy MM, Antonelli M, et al. (2017) Surviving Sepsis Campaign Guidelines. Crit Care Med 45: 486-552.

12. Rao SV, O'Grady K, Pieper KS, Granger CB, Newby LK, et al. (2005) Impact of bleeding severity on clinical outcomes among patients with acute coronary syndromes. Am J Cardiol 96:1200-1206.

Copyright: $\mathbb{C} 2020$ Stephens K. This is an open-access article distributed under the terms of the Creative Commons Attribution License, which permits unrestricted use, distribution, and reproduction in any medium, provided the original author and source are credited. 